\title{
Taller de cine y recursos audiovisuales para la recuperación en salud mental
}

\author{
Almudena Martínez de la Torre ${ }^{1}$, Irene Aranda Muros ${ }^{1}$, María Victoria Gómez Carracedo ${ }^{\text {, }}$ \\ Francisco Javier Durán Jiménez ${ }^{1}$, Pedro González Aceituno²
}

'Unidad de Rehabilitación de Salud Mental, Unidad de Gestión Clínica de Salud Mental, Hospital Regional Universitario de Málaga. Málaga, España. 2"Programa de intervención en salud mental y exclusión social", Unidad de Gestión Clínica de Salud Mental, Hospital Regional Universitario de Málaga. Málaga, España.

Contacto: amburdena@hotmail.com

Fecha de recepción: 16 de marzo de 2017 / Fecha de aceptación: 15 de julio de 2017

\section{Resumen}

Introducción: El objetivo de este escrito es dar a conocer un taller de creación cinematográfica para personas con trastorno mental grave (TMG) llevado a cabo en una unidad de rehabilitación de salud mental, basado en el modelo de recuperación y coordinado por enfermería de salud mental y un trabajador social.

Desarrollo: El taller se basa en la creación cinematográfica y la improvisación teatral. El proceso creativo parte de las ideas aportadas por el grupo relacionadas con situaciones variadas de carácter cómico habitualmente. Sobre esas ideas se hacen improvisaciones, que se graban y visualizan. Después se escogen aquellas ideas de mayor potencial y se definen unos guiones orientativos sobre los que seguir improvisando y grabando hasta configurar un corto.

Conclusiones: Poner en marcha grupos terapéuticos no convencionales según el modelo de recuperación puede obtener mejoras psicosociales en usuarios con TMG (creatividad, comunicación, autoestima, habilidades sociales, por ejemplo) y sirve como fuente de motivación profesional, sin suponer recursos costosos.

Palabras clave: terapia con arte, recursos audiovisuales, cine como asunto, rehabilitación, salud mental, recuperación, esquizofrenia.

\begin{abstract}
Title: Film Creation and Audiovisual Aids Workshop for Mental Health Recovery.

Objective: The aim of this text is to present a filmmaking workshop for people with severe mental disorder held in a rehabilitation unit of mental health, based on the recovery model and coordinated by mental health nurses and a social worker.

Development: This workshop is based on film creation and theatrical improvisation. The creative process comes from the ideas that the members of the group offer. The ideas are usually related to various comical situations. Improvisation pieces based on this ideas are recorded and visualised by the target group. The ideas with a greatest potential are used in order to create draft scripts that will be later developed. The final result is a short film.

Conclusions: Non conventional workshops in line with the recovery model can be positive for both users and workers, as workshops can be a motivation source that does not imply expensive resources. For users with severe mental disorder, this workshop involves improvements in psychosocial aspects (creativity, communication, self-esteem, social skills, for example).
\end{abstract}

Keywords: art therapy, motion picture as topic, audiovisual aids, recovery, mental health, rehabilitation, schizophrenia.

\section{Introducción}

Hace dos o tres años me di cuenta de que realmente era posible recuperarse.

La Unidad de Rehabilitación de Salud Mental del Hospital Regional Universitario de Málaga tiene por objeto la recuperación de habilidades sociales y la reinser- ción social y laboral, en régimen ambulatorio, de pacientes con trastorno mental grave (TMG).

La rehabilitación psiquiátrica se dirige a mejorar las capacidades de las personas con trastornos mentales en lo referente a su vida, aprendizajes, trabajos, socialización y adaptación de la forma más normalizada posible. Está basada en los propios esfuerzos de las perso- 
nas con TMG y es llevada a cabo por un equipo multiprofesional integrado por psiquiatras, psicólogos clínicos, trabajadores sociales, profesionales de enfermería y de terapia ocupacional y se trabaja en estrecha relación con los familiares ${ }^{2}$.

El enfoque de recuperación en salud mental se considera un nuevo marco conceptual en la rehabilitación moderna. Representa la convergencia de diferentes ideas (empoderamiento, autogestión, derechos de las personas con discapacidad, inclusión social y rehabilitación), bajo un solo encabezado que señala una nueva dirección para los servicios de salud mental ${ }^{3}$.

En concreto en personas con TMG, el modelo de recuperación conlleva que éstas sean capaces de desarrollar un proyecto de vida con sentido, independientemente de la evolución de sus problemas de salud, con el objetivo de que se alejen de la patología, la enfermedad y los síntomas, acercándose a la salud, la fortaleza y el bienestar. Desde este enfoque se estimula y facilita el manejo de uno mismo (autocontrol), para que se vaya consolidando una clara esperanza de recuperación y así descubrir un sentido de identidad personal separado de la enfermedad o discapacidad ${ }^{4}$.

En cuanto a los profesionales de salud mental, adoptar el modelo de recuperación como marco de trabajo conlleva cambios tanto en el rol profesional, como en la relación entre profesional y usuario/familia.

Las relaciones de ayuda entre profesionales, pacientes y familia pasan de ser una relación distante (el profesional en calidad de experto y el usuario en calidad de receptor pasivo) a ser una relación abierta, de confianza y honestidad, donde la calidad de la relación resulta crucial en la recuperación ${ }^{5}$. Esto implica una relación de poder diferente a lo habitual entre los profesionales y las personas que se atienden ${ }^{6}$. Hay que tener en cuenta que los profesionales, por sí solos, no pueden llevar a la práctica la recuperación. Esto sólo puede ser llevado a cabo por los usuarios y sus familias, pero pueden intentar crear condiciones en las cuales los individuos se sientan con poder de decisión, de tal modo que el sentido de la "autogestión" personal pueda desarrollarse . $^{3}$.

El modelo de recuperación es fácilmente aceptado por enfermería, por representar valores y competencias muy ligados a los cuidados, como la relación entre enfermera y paciente centrada en la persona, el enfoque holístico, el uso de la narrativa y el diálogo más natural, las relaciones basadas en la empatía y el calor humano, la promoción de la salud y la relación con personas usuarias en varios contextos, dentro y fuera de los servicios ${ }^{7}$.

Aunque las ideas de recuperación recibieron un fuerte impulso en los años 80 y suponen un principio organizativo en los servicios de salud mental de países como Nueva Zelanda, los Estados Unidos y Australia ${ }^{3}$, en nuestra unidad, es relativamente reciente la intención de adaptar los programas de intervención y nuestro papel profesional a este modelo.

Atendiendo a esta intención, surge la iniciativa de organizar y llevar a cabo el "Taller de cine y recursos audiovisuales para la recuperación en salud mental" que se va a describir para darlo a conocer. Dicho taller forma ya parte de los programas habituales que se ofrecen a los usuarios desde nuestra unidad. En el mismo también colabora personal que pertenece al Programa de Intervención en Salud Mental y Exclusión Social, en concreto un trabajador social.

La principal herramienta terapéutica que utilizamos para el taller es el cine como proceso creativo, es decir, desde la experiencia de crear cine y documentos audiovisuales. También entran en juego algunas dinámicas más relacionadas con técnicas teatrales como la improvisación.

\section{El cine y su uso como herramienta terapéutica}

\section{La recuperación me ha llevado a encontrar formas de devolver cosas a la gente que me importa. ${ }^{1}$}

Actualmente hay escasez de investigaciones sobre la eficacia del cine como herramienta terapéutica ${ }^{8}$ y más aún desde el proceso creativo y en el ámbito de la salud mental. La creación de una película o material audiovisual conlleva adquirir conocimientos, realizar decisiones a nivel grupal, ejercitar la capacidad de expresar ideas y sentimientos, generar un mensaje con propósito y reflexionar acerca de la temática representada con la intención de comunicar ${ }^{9}$. Además, se pueden identificar beneficios que pueden considerarse terapéuticos derivados de la participación en una actividad de este tipo, como pueden ser el desarrollo de la creatividad, la inteligencia, la percepción, el esfuerzo por objetivar, la capacidad de comunicación, de innovación, la resolución de problemas, el pensamiento divergente, la apertura al cambio, la observación, la experimentación, etc. $^{10}$ 
Por otra parte, se puede destacar el desarrollo de la ciudadanía a través de experiencias de creación de cine, ya que usando el cine como medio se pueden transmitir mensajes de concientización y conocimiento de diversas problemáticas de la sociedad desde la perspectiva de los usuarios, desarrollando a su vez valores y elementos de identidad y pertenencia?.

Otro beneficio de la creación cinematográfica radica en los recursos que le otorga a los profesionales que trabajan con personas con trastorno mental, ya que es una forma sutil y poco invasiva de obtener información acerca de los usuarios (sus sistemas familiares, relaciones interpersonales, creencias, valores, ideologías, expectativas personales, el concepto de sí mismo y de sus problemáticas...).

Teniendo esto en cuenta y que todas las personas tenemos capacidad de expresión artística, que incluyendo lógicamente a las personas con TMG, apostamos por esta iniciativa, apoyándonos en lo que se suele decir "la parte sana de la persona" y en métodos no convencionales para conseguir que construya un proyecto de vida.

Nuestra intervención a través del taller quedaría encuadrada dentro de las intervenciones psicosociales en personas con TMG, y dentro de éstas en las llamadas "terapias artísticas creativas", donde se incluyen entre otras: arteterapia, danzaterapia, biblioterapia, musicoterapia y dramaterapia ${ }^{11}$.

En el caso de la dramaterapia, que es la rama que más se acerca a nuestro taller, aún no se ha encontrado suficiente evidencia para efectuar recomendaciones de la misma en el tratamiento de $\mathrm{TMG}^{12}$, aunque sí se han observado beneficios positivos de las terapias artísticas creativas en general ${ }^{13}$.

Además, la arteterapia está reconocida como intervención enfermera, al estar incluido en la Nursing Intervention Classificaction (NIC) como "Terapia Artística" (4330), definida como "la facilitación de la comunicación por medio de dibujos u otras formas de $\operatorname{arte}^{, 14}$.

En el taller se trabajan diferentes objetivos, relacionados con los usuarios, con los cuidados enfermeros y con la sociedad en general. Lo que se pretende mejorar en los usuarios son aspectos como autoconciencia, expresión, comunicación, relaciones interpersonales, autoestima, confianza en uno mismo, creatividad, habilidades sociales, crecimiento personal y social a través de experiencias positivas, y la búsqueda de la ilusión por la vida para desarrollarla con sentido. En cuanto a los cuidados enfermeros se abordan diagnósticos enfermeros como "Baja autoestima crónica" (00119), "Desesperanza" (00124), "Deterioro de la interacción social" (00052), "Aislamiento social" (0053) y "Riesgo de Soledad" (0054) ${ }^{15}$, utilizando la creación de material audiovisual como herramienta terapéutica.

Los objetivos para la sociedad en general van dirigidos a luchar contra el estigma en salud mental al fomentar una visión positiva de las personas con TMG, haciendo visible su aportación a la sociedad a través de la proyección de producciones creadas.

\section{Desarrollo}

Me he convertido en dueño de mi enfermedad y asumo la responsabilidad de lo que hago y de lo que no hago. ${ }^{1}$

Para dar a conocer el taller creemos importante explicar los orígenes y el desarrollo en el tiempo del mismo y así que se entienda cómo hemos llegado a la organización y funcionamiento actual.

El taller se empieza en octubre de 2013 en la unidad de rehabilitación después de algunas experiencias anteriores, en las que se realizaron cortos concretos y esporádicos. Como se vio el efecto positivo en las personas que participaron, nos propusimos instaurarlo de manera permanente, como una actividad estructurada, con el objetivo de diversificar las actividades terapéuticas, y que tuviera como eje central la diversión.

En una primera etapa nos pusimos a elaborar un guion, pero fue algo difícil de hacer en colectividad. Resultaba una tarea aburrida, la participación era escasa y estábamos obteniendo un resultado contrario al pretendido.

Pasamos a otra fase, abriendo el taller también a usuarios de las unidades de salud mental comunitarios, que son personas con un mayor nivel de autonomía. En el mismo, cada participante planteaba en las sesiones pequeños sketches que representábamos y grabábamos para ser visionados al final. Esta experiencia facilitó la creatividad del grupo. Cada cual aportaba al guion su impronta, sus ocurrencias (sin ningún miedo a salirse del guion), las aportaciones durante las grabaciones, el diálogo espontáneo, las complicidades generadas..., 
facilitando que el objetivo terapéutico se viese más nítido. Posteriormente, con la proyección en el grupo de las grabaciones, en las que los participantes veían sus gestos, sus expresiones y su creatividad, disfrutando y con satisfacción, empezamos a ver el valor del audiovisual como herramienta terapéutica.

En enero de 2014, nos propusimos dar otro paso: realizar el taller fuera del ámbito sanitario y dejar de lado los roles de profesionales sanitarios, pacientes y familiares, considerándonos todas las personas participantes igual de neófitos en el tema y con la necesidad de aprender los unos con los otros. El desarrollo de la recuperación se apoya en las cualidades personales de los profesionales, al mismo nivel que en su formación académica (uno de los principios de la recuperación). Con esta orientación el taller se empieza a desarrollar en la sede de la Asociación Arrabal (organización de Málaga que trabaja por la incorporación laboral y social de personas desempleadas en riesgo de exclusión social), en un intento de des-psiquiatrizar aquello que no tiene sentido psiquiatrizar.

Una vez en Arrabal, podríamos decir que "normalizando" la realización del taller en un contexto social, se avanza abriendo el taller a personas ajenas a nuestro trabajo. En un intento de luchar contra el estigma asociado a la enfermedad mental no aceptamos que los talleres, aunque tengan como objetivo prioritario una función terapéutica, deban ser solo y en exclusiva para personas con enfermedad mental.

El objetivo principal de este taller es terapéutico, pero ello no implica que todas las personas que participan en él sean personas con TMG.

Es un taller abierto a la participación de quienes quieran vivir esta experiencia, tanto del ámbito de salud mental como de la sociedad en general. En este tiempo se han incorporado estudiantes de pregrado (Psicología, Enfermería, y Trabajo Social), especialistas de salud mental en formación postgrado (Enfermería de Salud Mental, Psicología Clínica y Psiquiatría), un familiar y colaboradores puntuales del ámbito audiovisual. Está coordinado por un enfermero especialista en salud mental y un trabajador social.

El proceso de recuperación está muy relacionado con los procesos de inclusión social y con la capacidad de disfrutar de un rol social, de ahí la importancia de la participación de personas diversas, no solo del ámbito sanitario y no solo personas con trastorno mental.
En la actualidad, en el grupo participan unas ocho personas de forma estable, además de estudiantes, familia y colaboradores. La propia dinámica del grupo permite la entrada y salida abierta de participantes esporádicos. Cabe destacar aquí la sorpresa al descubrir el potencial de expresión artística e interpretativa de muchos de los participantes, que no dudan en tomar la iniciativa y adoptar un papel activo.

El taller se realiza una vez a la semana, con una duración de dos horas, en el que las diversas sesiones van conformando el proceso creativo, siempre con la flexibilidad y adaptación al ritmo del grupo, que será el que irá marcando el progreso. No hay guion previo. Las ideas son lanzadas al grupo por cualquiera de los participantes. A partir de ellas, mediante asociaciones y tormenta de ideas, se va enriqueciendo y debatiendo aquello sobre lo que se puede trabajar, hasta que queda la idea de un sketch básico (escena, generalmente humorística, que dura entre uno y diez minutos aproximadamente) como punto de partida. Por lo general se suelen incluir juegos de palabras en los sketches que dan pie a confusión y a situaciones cómicas.

El siguiente paso es la representación libre de lo que se ha acordado como idea básica o situación de partida (a modo de consigna para comenzar la improvisación), pero se improvisa todo lo demás: el diálogo, la escenificación, la interacción entre actores..., ya que damos mucho valor a la creatividad de cada una de las personas participantes. Según la idea básica o situación de partida, entran a escena dos o más participantes y representan su versión improvisada de dicha idea, de modo que todos tienen la oportunidad de desarrollar y representar lo que la situación de partida les sugiere en cada sesión. Estas representaciones son grabadas por una cámara de video y visualizadas en la última fase de la sesión, algo que da pie a nuevas ideas y reflexiones debatidas en grupo. En este momento del proceso creativo, cada sesión consta de un periodo inicial de improvisaciones, en el que todos, incluidos profesionales, estudiantes, colaboradores y familiares, participan, y una posterior visualización de lo grabado.

Durante varias sesiones se sigue trabajando el mismo sketch. Los papeles se intercambian, se producen nuevas aportaciones, se sigue visionando lo grabado al final de la sesión y se va perfilando la estructura definitiva del material audiovisual a realizar. Por lo general, se orienta el resultado a la creación de un cortometraje. 
Cuando ya se tiene claro qué queremos representar, se fijan los papeles y se escriben unos guiones orientativos con varias escenas surgidas de la improvisación, procediéndose nuevamente a la grabación que formará parte definitiva del corto. El texto del guion es solo orientativo, no es preciso que ningún participante lo memorice. Durante la grabación, todos sabemos cuál es la estructura, las escenas y las situaciones de partida porque se ha venido trabajando y reflexionando en sesiones anteriores, pero se producen nuevas improvisaciones. Lo que se obtiene de algún modo es que las ideas, guiones y contenidos se interiorizan desde la asistencia a las sesiones sin que sea necesario dedicarle mucho tiempo o esfuerzo fuera de las mismas. Previo a este momento también se han podido decidir lugares de grabación o incluso vestuario si procede.

La aceptación de todas las ideas, la flexibilidad, y la improvisación que caracterizan al taller hacen posible que todo aquel que asiste, aun no siendo constante en la asistencia, pueda participar sin la presión de tener que cumplir unas expectativas de participación programada.

El rol que juega enfermería en el desarrollo del taller es clave, ya que actúa dinamizando, coordinando y motivando a los participantes, además de organizar sus propuestas. Siempre lo hace desde la perspectiva de la recuperación, poniendo en juego valores que tienen que ver con la misma como el estilo empáticoparticipativo, el enfoque humanista, el empoderamiento...

Posteriormente hay un proceso de editado y montaje de las distintas grabaciones. Para ello solemos contar con la colaboración de voluntarios con conocimientos técnicos en comunicación audiovisual. Esto hace posible la creación de un documento audiovisual o cortometraje que puede ser proyectado en función de los intereses del grupo. Hay que destacar aquí que lo fundamental es el proceso de creación, no tanto el resultado, aunque las experiencias hasta ahora han sido favorables, con resultados muy aceptables dentro del mundo actual de la creación audiovisual.

La proyección también conlleva la emoción y satisfacción de mostrar un trabajo bien hecho a otras personas, aumentando la valía y autoestima de los que participan, así como también es una oportunidad de participación en la sociedad y de que se reconozca el papel activo que las personas con TMG pueden tener como cualquier otra.
A día de hoy se han realizado dos cortos cuyos títulos son Invisibles, que ha sido proyectado en diversos eventos relacionados con la salud mental, y Sin Lugar, aún por proyectar. Está en fase de montaje el tercero, titulado Dejardinero, aunque no podemos olvidar experiencias de improvisación anteriores.

Como consideraciones éticas, decir que previamente obtenemos el consentimiento informado y escrito de todos los participantes para la grabación y proyección del resultado. Y además se tiene en cuenta lo previsto en la Ley 41/2002 de 14 de noviembre, básica reguladora de la autonomía del paciente y de derechos y obligaciones en materia de información y documentación clínica y lo previsto en la Ley Orgánica 15/1999, de 13 de diciembre, de protección de datos de carácter personal.

En cuanto a lo que ha aportado el taller a las personas, subrayamos que no se puede uniformizar. El proceso ha sido similar para todos, pero funciona de manera individualizada y diferente en cada persona, aunque a todas las personas participantes nos ha aportado algo positivo, sea persona con $\mathrm{TMG}$, familiar, estudiante, colaborador o profesional de la salud mental.

Por señalar solo aquellos aspectos en los que más hemos observado cambios positivos entre las personas con TMG que han participado, podemos nombrar los siguientes:

- Creatividad: Realizan con mayor fluidez las asociaciones de ideas, responden con agilidad a las intervenciones de las otras personas, aportando riqueza a los diálogos.

- Comunicación verbal: La pobreza y pérdida de fluidez en la expresión verbal ha mejorado.

- Comunicación no verbal: Existen dificultades motoras, por la enfermedad y como efecto secundario de la medicación. La expresividad se ha visto beneficiada.

- Autoestima y confianza en sí mismos: Se intuye desde la observación que ha mejorado al ver su entusiasmo e ilusión al sentirse protagonistas de una obra que han elaborado desde su inicio: ideas, estructura del sketch, enriquecimiento de los diálogos e interpretación.

- Concentración: Se ha visto mejor capacidad ya que el taller requiere estar pendiente del desarrollo del trabajo grupal.

- Interacción y habilidades sociales: El aislamiento social propio de la enfermedad mental se rompe y se 
observa una mejora importante, más cuando empezamos a participar los profesionales sanitarios como iguales y aún más cuando se incluyen en el taller a personas allegadas que no pertenecen al ámbito sanitario.

- Reconstrucción de la imagen corporal: Verse en la pantalla ha facilitado una capacidad autocrítica para mejorar su imagen, tanto en la forma de vestir, como en lo corporal. En general estaban sorprendidos al verse ("¿Yo soy ese?", "No me reconozco"...).

- Atención: Es una dificultad asociada a la enfermedad mental y que mediante el taller ha ido mejorando, ya que es fundamental para poder responder a las improvisaciones de los demás.

\section{Conclusiones}

\section{Nada te debería impedir ser lo que quieres ser... Nunca dejes que nadie te lo diga.}

Considerando que el enfoque hacia el modelo de recuperación se prevé que sea el futuro de la rehabilitación en salud mental, queda justificada la intención de adaptar nuestros programas de intervención enfermera y de crear nuevos programas orientados a dicho modelo.

Como resultado de ello, organizamos en nuestra unidad el taller que se ha presentado, que además de haber obtenido beneficios y resultados terapéuticos observables en nuestros pacientes, también ha posibilitado una aproximación de nuestra intervención como enfermeras a una serie de principios en los que se basa el modelo de recuperación. Como ejemplo destacamos: relación entre profesional y paciente alejada del formato profesional experto-paciente pasivo, participación de familiares, allegados y más personas de la sociedad civil, uso de espacios no sanitarios, programa basado en cualidades y habilidades del profesional... Estos aspectos son importantes para favorecer la inclusión social, principal objetivo de nuestras intervenciones en salud mental, más que la cura.

En cuanto a las proyecciones de los resultados del taller en forma de cortometrajes cinematográficos, decir que es difícil de valorar el impacto en las personas que han podido disfrutar de ellas, pero sí podemos asegurar que hasta ahora no han dejado a nadie indiferente.
Otro aspecto a destacar es lo productivo que resulta a nivel profesional la puesta en marcha de programas de intervención para la rehabilitación en salud mental no convencionales, como fuente de motivación y creación de vínculo terapéutico.

Por último, comentar que serían necesarios estudios específicos para evaluar la eficacia de la intervención enfermera con grupos/talleres terapéuticos con orientación a la recuperación, dirigidos a pacientes con TMG. Esto resulta importante para contar con suficiente evidencia científica para recomendar nuestro taller o experiencias similares en el tratamiento del TMG. Puede ser útil utilizar la Clasificación de Resultados de Enfermería (NOC) ${ }^{16}$ para objetivar la evolución positiva en determinados aspectos ya mencionados en los usuarios que asisten al taller y que se relacionan con diagnósticos enfermeros comunes en TMG.

A pesar de no contar con evidencia científica firme para recomendar el taller presentado, no hemos de olvidar que se han observado beneficios positivos en la salud mental a través del uso de diversas terapias artísticas creativas ${ }^{13}$, por lo que está más que justificado su uso.

\section{Agradecimientos}

Se agradece a la AEESME la publicación de este manuscrito, el cual se realiza después de haber recibido el $2^{\circ}$ premio la comunicación oral en la que se basa su redacción, en el XXXII Congreso Nacional de Enfermería de Salud Mental y II Conferencia Internacional de Enfermería de Salud Mental celebrados en mayo del 2015 en Burgos.

\section{Bibliografía}

1. Brown W, Kandirikirira N. Recovering Mental Health in Scotland. Scottish Recovery Network, Baltic Chambers, Suites 2007; 50 : 320-332.

2. Anthony WA, Liberman RP. The Practice of Psychiatric Rehabilitation. Schizoph Bull 1986; 12: 542-59.

3. Shepherd G, Boardman J, Slade M. Making Recovery a Reality. Sainsbury: Sainsbury Centre for Mental Health, Policy Paper; 2008.

4. Davidson L. Recovery Concepts and Application. Devon Recovery Group 2008.

5. Roberts G, Wolfson P. The rediscovery of recovery: open to all. Adv Psychiatr Treat 2004; 10: 37-49.

6 . Repper J. Perkins R. Social Inclusion and Recovery. London: Balliere Tindall; 2003.

7. Caldwell, Barbara A; Sclafani, Michael; Swarbrick, Maragret; Piren Karen. Psychiatric nursing practice \& de recovery model of care. Journal of Psychosocial Nursing. 2010; 48(7): $42-48$ 
8. Armijo N. Uso de la Cineterapia en Mujeres Víctimas de Violencia Intrafamiliar. Tesis para optar al grado de magíster en Psicología de adultos. Universidad de Chile: 2010

9. Cancino González PM. El cine y su uso como herramienta de tratamiento en terapia ocupacional. Revista Chilena de Terapia Ocupacional 2013; 13(1):51-57.

10. Granados IM. Interrelaciones entre creatividad, arte, educación y terapia. Revista electrónica de la Universidad de Jaén: Arte y movimiento 2009; 1:51-62. Disponible en URL:

http://webcache.googleusercontent.com/search?q=cache:ZWv t3KEJj5wJ:revistaselectronicas.ujaen-

.es/index.php/artymov/article/download/144/132+\&cd=3\&hl=e $\mathrm{s} \& \mathrm{ct}=\mathrm{clnk} \& \mathrm{gl}=\mathrm{es}$

11. Landy R. The future of drama therapy. The Arts in Psychotherapy 2005; $1-8$.

12. Grupo de Trabajo de la Guía de Práctica Clínica de Intervenciones Psicosociales en el Trastorno Mental Grave. Guía de Práctica Clínica de Intervenciones Psicosociales en el Trastorno Mental Grave. Plan de Calidad para el Sistema Nacional de Salud del Ministerio de Sanidad y Política Social. Instituto Aragonés de Ciencias de la Salud-I+CS; 2009. Guías de Práctica Clínica en el SNS: I+CSN²007/05

13. Rodríguez Barquero, V. El mejoramiento de la salud a través de la expresión corporal: un enfoque holístico. Revista Reflexiones 2008; 87(1): 127-137. Disponible en URL:

http://www.redalyc.org/articulo.oa?id=72912553009

14. Bulechek GM, Butcher HK, Dochterman JM, Wagner CM, editores. Clasificación de Intervenciones de Enfermería (NIC). $6^{\mathrm{a}}$ ed. Madrid: Elsevier; 2014

15. NANDA Internacional. Diagnósticos enfermeros: definición y clasificaciones 2012-2014. Madrid: Elsevier; 2012.

16. Moorhead S, Johnson M, Maas ML, Swanson E, editoras. Clasificación de Resultados de Enfermería (NOC). Medición de resultados

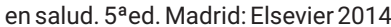

